\title{
Design of Construction Project Management Information System Based on CPR Theory
}

\author{
Cui Bo \\ State Key Laboratory of Hydraulic Engineering Simulation and Safety, Tianjin University \\ Tianjin, China 300072 \\ cuib@tju.edu.cn
}

Keywords: CPR; construction project; management information; system design

\begin{abstract}
Construction process reengineering (CPR) is used to reengineer the construction process of engineering project by using the thought and method of business process reengineering (BPR) to improve the efficiency of engineering construction. CPR theory provides improvement direction for construction project information management: On the data-oriented and function-oriented bases, it can realize the support of engineering construction project management information system for management process, and realize the objective of information integration and knowledge sharing under the premise of process optimization. The paper makes research on the process and method of construction project management process reengineering based on CPR theory, puts forward design thought for engineering construction project management information system based on CPR theory, and uses J2EE technology to set up information platform to realize the support for construction management process.
\end{abstract}

\section{Introduction}

The implementation of modern construction engineering project is a complex system composed jointly by the multiple units and a number of departments; all participants have to standardize the information process between them and organize the reasonable information flow to achieve communication at any time. With the emergence of construction process reengineering (CPR) theory, modern engineering construction project management information system has to face the whole process of construction process reengineering not only to implement the results of the process reengineering to support the new operation manner of the project management process, but also put itself in the changing environment of the process reengineering.

Construction process reengineering is the application of business process reengineering (BPR) in engineering construction management field as the new concept. For research on CPR, three typical process reference models were appeared in 1980s and 1990s [1]: "the RIBA Plan of Work", "the BPF manual”, "the Generic Design” and "Construction Process Protocol”. For idealization situation of macroscopic level, these models cannot be implemented specifically and effectively in the whole construction process of engineering project. After entering the 21st century, Cheng, et al used construction management process reengineering (CMPR) method to improve the efficiency of construction management in combination with BPR theory in 2003, and defined four steps of CMPR method: Process description, process conversion, process evaluation and reengineering behavior [2]; They put forward team human resource plan (THRP) to configure the human resource based on this in 2006 [3]. The two studies are restricted to management level of construction enterprise, and fail to explain the application of CPR theory in modern engineering construction projects management with numerous participants and complex process. Based on the theory of CPR, the paper is to discuss the process and method of construction project management process reengineering, and research on engineering construction management information system based on management process under network environment. 


\section{Reengineering of construction process}

Construction process reengineering (CPR) is used to reengineer the construction process of engineering project by using the thought and method of business process reengineering (BPR), namely the extension and application of engineering construction field. Mohamed S. defines the concept of CPR as follows: "A complete set of methods, with the owner as the core, to gradually build up integration project construction process, aims at optimizing foreseeability in project process and value-added to the final construction products" [4]. The enlightenment of this definition to the information management of engineering construction project is to establish a mode that facilitates the owner, project management party, designer, construction party, supervisor and supplier to extensively participate in the project process. Based on the support of integrated information platform, under the premise of process optimization, realize the objective of information integration and knowledge sharing to optimize the engineering cost, quality, construction period and other key performance.

Reengineering of construction process is constituted of the following five steps [5]:

(1) Preparation of process reconstruction. Including: Establish specialized team across the participant: Formulate process objective of reengineering of process, and combine consideration of external environment impact on the basis of fully considering the owner demands; Select the process that needs to be reengineered, mainly those core and normalized construction management process with low efficiency due to organization and geographical boundary under traditional organization mode and information communication cooperation manner.

(2) Analysis of original process. Including: Establish original process model; Analyze and evaluate the original process, and summarize time and cost that all activities consume based on the knowledge of activities of original process; Identify non-value-added and value-added activities on the basis of above analysis evaluation.

(3) Design of new process. Including: Analyze and compare relative optimal process of other organization in original process and similar conditions to acquire improved thoughts; Redesign the original process based on process design principle, combined with above analysis and comparison results to establish feasible and new process model; evaluate new process and select optimal scheme.

(4) The implementation of new process. Including: Formulate specific implementation plan according to optimal scheme, and reform organization structure and culture accordingly; Map new process model to workflow management module of information system to realize new business process automation supported by computer; Conduct necessary education and training to related personnel

(5) Ongoing improvement. Including: Constantly evaluate implementation achievement and establish necessary feedback mechanism; continuously optimize and improve during operational process.

It needs to point out that, during construction process, the business process shall include professional work process and construction project management process implemented by construction project. Professional work process is aimed at construction products, whose implementation ensures procedure and rationalization of project implementation process and the completion of engineering construction task, and reflects objective law of construction production. Project management process organically combines information flow and material flow required by construction project management to ensure reasonable coordination between professional engineering implementation and department, and reflect characteristics of project management activities. The design of construction project management information system is based on the project management process.

Five steps of reengineering of construction process are end to end and circulated in the overall process of construction management, where, establishment of optimized process model is more important. For the construction project management information system based on management process, analysis of workflow of new process and construction of workflow suitable for computer are the key of systematic design success. In addition, implementation of project management work and fulfillment of related management function are requested to determine detailed functional 
management tasks, and implement the management tasks to personnel and department. Therefore, information system design shall be based on management of work process and related organization as well as the corresponding management responsibility.

The successful implementation of a construction project is guaranteed by a series of coherent and interlaced management process, which is closely related. The separate existence after the improvement of single process or partial functions without consideration of overall coordination is meaningless. Construction process integration is one of the objectives of CPR [5]. Therefore, it is necessary to analyze and evaluate and modify each process with the idea of system integration, and try to realize the rationality, purpose and overall coordination of the process.

\section{Construction project management information system design process based on CPR theory}

Based on the CPR theory, the construction project management information system design makes a reasonable plan on the bases of construction management process reengineering, workflow system design and management process integration, determining which steps can be canceled, merged or further decomposed, to achieve dividing functional modules with the purpose of project management objectives, establish a new system function model, and carry out code design, flow chart design, functional structure chart design, input and output design and processing design, etc.. It is mainly divided into the following phases:

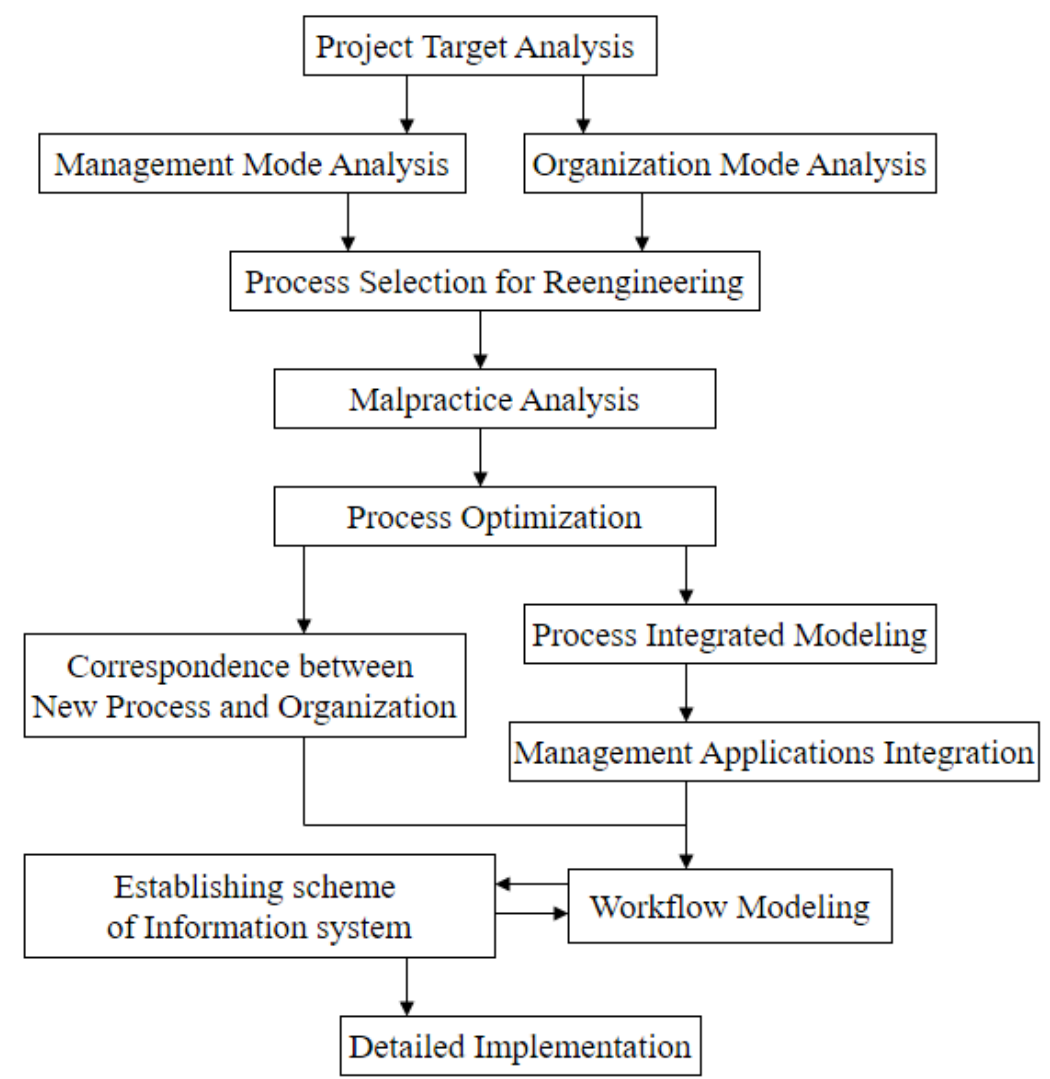

Fig. 1 design process of management information system

Phase I: Objective analysis of construction project management. The work of this phase is to understand the management objectives of construction projects, understand the management mode and organization mode of the project in a detailed way, and select the management process that needs restructuring, in order to ensure that the project management objectives remain consistent with information system construction objectives.

Phase II: Management process reengineering of construction project. The work of this phase is to fully understand the operation manner of the management processes and their sub-processes, analyze the existing problems in the current management process, and improve the original process, propose a new process plan according to the project objectives and the potential of information technology. 
Phase III: Management process integration of construction project. The work of this phase is to put forward the adaptive organization management mode based on the optimized management process, establish a new responsibility distribution matrix, and achieve the integration of management work and organization; At the same time, realize the integration of project management application based on the integrated management process modeling.

Phase IV: Workflow system design. The work of this phase is to conduct modeling through the workflow based on the reengineered and integrated management process, transform the management process into the workflow the computer can identify, and realize the automation of the new management process that the computer supports.

Phase V: Put forward the construction scheme of management information system, make the implementation plan and detailed rules of the scheme and determine the specific development method of the subsystems.

Design process of management information system is shown in Figure 1.

\section{Case study}

A hydropower engineering is located on the Panlong river of Yunnan Province. The construction scale of the engineering is large, the construction condition is complex, and many factors are involved, which bring some difficulties to the engineering construction management. It is necessary to strengthen the information management of engineering construction to improve the level and efficiency of engineering construction management. Through careful field investigation, master the actual management work process of the engineering, use the idea of process reengineering to optimize the management process, so as to realize the integration of a number of management applications. On this basis, apply J2EE technology, use the most advanced three-layer logic structure (application layer, service layer and data layer), and use multi-level distribution system structure to establish the integrated management information system platform of the engineering project (as shown in Figure 2).

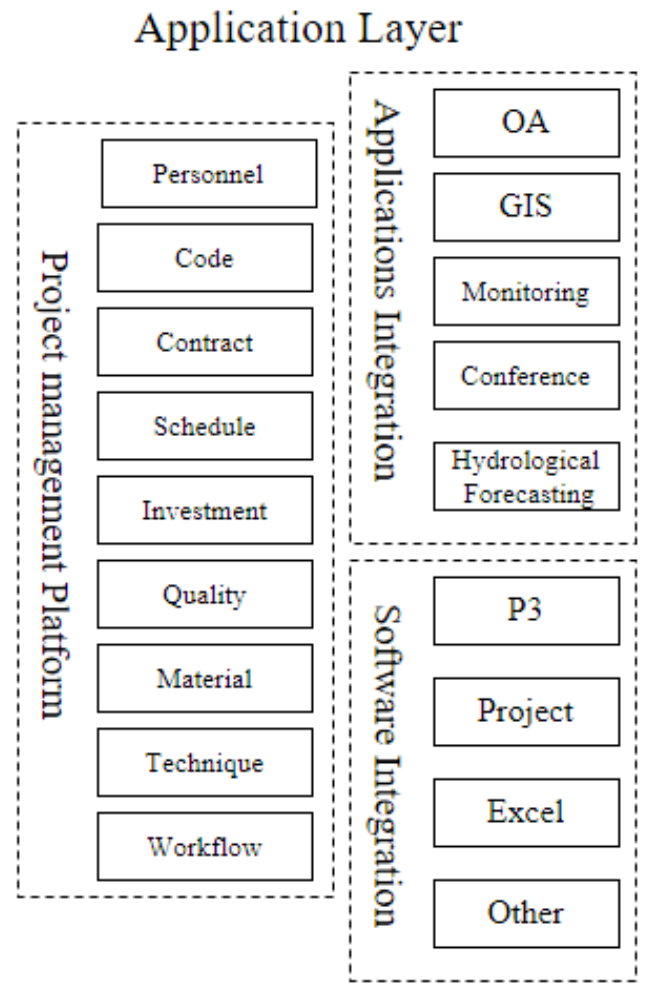

Service Layer

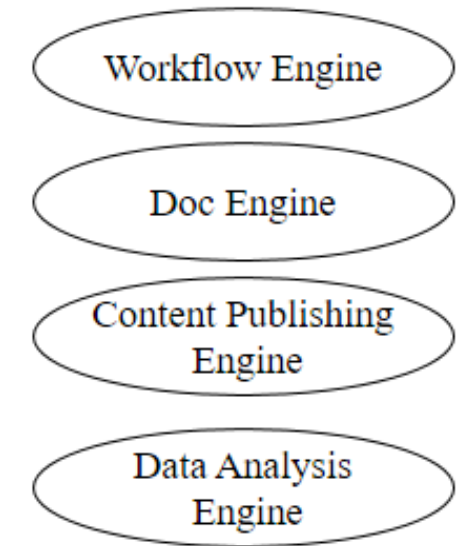

Application Integration

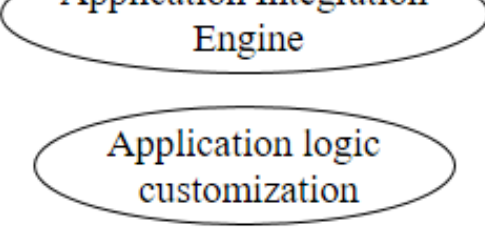

Data Layer
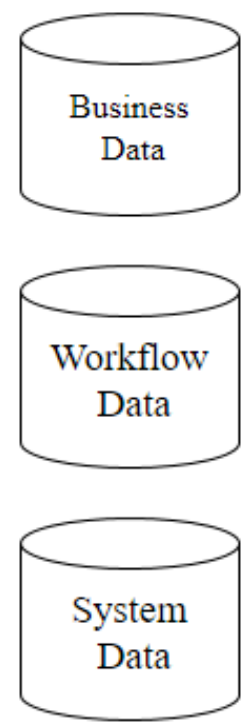

Fig. 2 the three-layer logic structure of the integrated management information system platform of the

(1) Application layer engineering project 
While the application layer provides project management interface, it integrates a variety of engineering management software and heterogeneous application systems, to achieve diversity, characteristics and sharing of the project construction management information. Each functional subsystem realizes the information communication through the encoding system; the integrated information platform provides the interface to realize the software application integration and the heterogeneous system information integration.

Workflow management in application layer includes process definition tool, message reminder tool and workflow monitoring tool, which realizes the application function through the access to the business logic layer in workflow engine. The process definition tool is used to provide a graphical process definition tool for process management personnel; Message reminder tools provide the message reminder function of to-do list for the participants in the workflow; Workflow monitoring tool provides a means for the workflow management personnel to monitor the operation of the workflow, examine the operation status and the workflow resource management.

(2) Service layer

As a system support platform, the service layer is mainly used for the customization of various application logics, so it is also known as the business layer. Business layer can achieve specific business logic and database storage mainly through EJB (Enterprise JavaBeans) and other technologies. EJB can receive data from the client application procedure and send data to the data layer for storage after processing the data; On the other hand, EJB can retrieve data from the data layer and send the results to the client application procedure.

In the customization of various application logics (such as the design of workflow engine), generally it is necessary to realize the mapping of the data in the data layer on the business logic object, namely, establish a data access layer to provide a unified data access manner for business logic. Data access layer uses the entity Bean manner to encapsulate the operation of data, so that the business logic can be separated from data source, which is easy for expansion.

In the workflow engine, the business logic that can realize interpretation, execution, control, and other business operations service of the workflow reads and writes data layer through data access layer. Realize the business logic by using the session Bean+ aided manner. While realizing the internal function of the workflow engine, provide access and management interface for the external application, and realize the interaction between each kind of application of the application layer and workflow management system.

(3) Data layer

Data layer is the data base of management information system, which is composed of database and document management system. Where, the database, in a unified way, saves and manages the project management application data, the workflow management data, the organization data and the system operation data. And the document system supports database to save data of the document type.

\section{Summary}

Based on workflow technology, this paper studies the information integration of engineering construction project based on management process. On one hand, realize integration of management work and organization by researching the integration method of management work breakdown structure (MWBS) and organization breakdown structure (OBS); On the other hand, research the integrated management method of management process, apply IDEF0 method for integrated modeling of management process, and build integrated framework of management work based on workflow, so as to realize the integration of information system manner of management process. The research results can provide the theory and method support for the information integration of engineering construction project. 


\section{Acknowledgements}

This work is financially supported by the National Natural Science Foundation of China (No. 51209159), Tianjin Natural Science Foundation (No. 13JCQNJC08900) and the National Basic Research Program of China (973 Program) (No. 2013CB035906).

\section{References}

[1] Margaret N, Angela L, Rachel C, et al.

http://www.processprotocol.com/pdf/cobrapaper1999.pdf

[2] Min-Yuan Cheng, Min-Hsiu Tsai. Journal of construction and management, ASCE, 2003, 129 (1)

[3] Min-Yuan Cheng, Ming-Hsiu Tsai, Zhi-Wei Xiao. Automation in construction, 2006, 15

[4] David Thorpe. Fifth annual conference innovation in construction, Brisbane, Australia, 2000.11

[5] Lu Yong. Infrastructure Optimization, 2005, 3 (3) 